\title{
Los Influencers Virtuales como herramienta publicitaria en la promoción de marcas y productos. Estudio de la actividad comercial de Lil Miquela
}

\section{Virtual Influencers as an advertising tool in the promotion of brands and products. Study of the commercial activity of Lil Miquela}

Luis Rodrigo-Martín. Universidad de Valladolid. España.

luis.rodrigo@uva.es

$[\underline{\mathrm{CV}}]$ (1) G $\mathrm{R}$

Isabel Rodrigo-Martín. Universidad de Valladolid. España.

isabel.rodrigo@uva.es

$[\mathrm{CV}](\mathrm{G}) \mathrm{C}$

Daniel Muñoz-Sastre. Universidad de Valladolid. España.

daniel.munoz.sastre@uva.es

$[\mathrm{CV}] \bigcirc \mathrm{G}$

Cómo citar este artículo / Referencia normalizada

Rodrigo-Martín, L., Rodrigo-Martín, I. y Muñoz-Sastre, D. (2021). Los influencers virtuales como herramienta publicitaria en la promoción de marcas y productos. Estudio de la actividad comercial de Lil Miquela. Revista Latina de Comunicación Social, 79, 69-90. https://www.doi.org/10.4185/RLCS-2021-1521

\section{RESUMEN}

Introducción: En los últimos años se ha producido una progresiva e intensa migración de audiencias entre los medios de comunicación tradicionales y las nuevas fórmulas digitales, especialmente las redes sociales. Especialmente entre los sectores más jóvenes de la población y que, por tanto, presentan un perfil más atractivo como consumidores para los anunciantes, esta tendencia se agudiza. Esta modificación del panorama mediático exige la implementación de nuevas estrategias comunicativas y publicitarias por parte de los anunciantes. El auge de los denominados influencers ha supuesto una verdadera novedad a la hora de dar a conocer y aumentar el deseo consumo de marcas y productos de diferentes sectores. Metodología: Para llevar a cabo nuestro estudio hemos empleado una metodología multimétodo que consta principalmente del uso de la investigación científica cualitativa y cuantitativa para conseguir analizar estas estrategias comunicativas y sus posibilidades comerciales en el actual contexto digital. Resultados: El objeto de la presente investigación consiste en analizar el papel de los denominados influencers virtuales, avatares creados mediante técnicas de inteligencia artificial y realidad aumentada que permiten aunar las posibilidades comunicativas y publicitarias del influencer tradicional con el control absoluto de su actividad y 
"vida" en las redes. Discusión: Los influencers se han convertido en los líderes de opinión del siglo XXI y las marcas comerciales apuestan por ellos para lanzar sus mensajes. Conclusiones: Los influencers virtuales son un elemento necesario para entender la evolución de la comunicación publicitaria en el actual contexto digital.

PALABRAS CLAVE: Influencers; marca; comunicación; prescripción de producto; redes sociales; publicidad; Instagram.

\begin{abstract}
:
Introduction: In the last years there has been a progressive and intense migration of audiences between traditional media and new digital formats, especially social networks. This trend is becoming more acute, especially among the younger sections of the population who are therefore more attractive to the population who are therefore more attractive to advertisers as consumers. This change in the media landscape requires the implementation of new communication and advertising strategies by advertisers. The rise of the so-called influencers has been a real novelty when it comes to publicizing and increasing the desire to consume brands and products from different sectors. Methodology: To carry out our study, we used a multi-method methodology that mainly consist of the use of qualitative and quantitative scientific research to analyse these communication strategies and their commercial possibilities in the current digital context. Results: The aim of this research is to analyze the role of so-called virtual influencers, avatars created by artificial intelligence and augmented reality techniques that allow the traditional influencer's communication and advertising possibilities to be combined with the absolute control of his activity and "life" on the networks. Discussion: Influencers have become the opinion leaders of the $21^{\text {st }}$ century and commercial brands are betting on them to deliver their messages. Conclusions: Virtual influencers are a necessary element to understand the evolution of advertising communication in the current digital context.
\end{abstract}

KEYWORDS: Influencers; brand; communication; product prescription; social media; advertising; Instragram

\title{
CONTENIDO
}

1. Introducción. 2. Objetivos. 3. Metodología. 4. Discusión/Resultados. 5. Conclusiones. 6. Bibliografía 7. Currículum Vitae

\section{Introducción}

El auge de las redes sociales entre los sectores más jóvenes de la población ha permitido que se conviertan en un elemento importante en su forma de comprender el mundo que les rodea. Una vez superadas las percepciones diferenciadas entre el mundo real y virtual, las redes sociales se han convertido en un ámbito más en el que las personas se relacionan, interactúan y conviven. "La habilidad que algunos menores están demostrando para generar contenido (UGC) gestionarlo y crear una comunidad de seguidores suscita el interés de las marcas que aspiran a comunicar de forma empática" (Tur-Viñes et al, 2018). Tomando esto en consideración, no es de extrañar que estos nuevos medios se hayan convertido en un elemento estratégico relevante para los anunciantes tradicionales que encuentran en las redes sociales una nueva oportunidad para conectar con audiencias crecientes a las que cada día es más complicado llegar a través de otros medios más convencionales. Según estudios preliminares, los jóvenes, debido a su inocencia y autoconfianza, son el sector más activo en redes sociales siguiendo perfiles que reflejan estilos de vida similares a los suyos (González et al., 2021) Las lógicas de participación en estos modelos de interacción social exigen nuevos replanteamientos creativos y estratégicos (Mañas-Viniegra, L. y Jiménez-Gómez, I., 2019) en tanto que se configuran como espacios de seguimiento y participación en un plano de 
igualdad. A diferencia de lo que sucedía en los planteamientos clásicos de la publicidad en la que el medio imponía los contenidos que se consumían sin posibilidad alguna de elección por parte de las audiencias, las redes requieren un esfuerzo de integración para los anunciantes a los que los usuarios seguirán, o no, en función del atractivo que consigan generar. Por todo ello es necesario innovar en la construcción de perfiles sugerentes, con actividad cotidiana, que aporten contenidos relevantes en el fondo y en la forma y que interactúen en un plano de igualdad y respeto con otros usuarios de las redes.

El mecanismo más utilizado para acelerar el proceso de creación de un perfil con seguidores suele ser la colaboración con influencers, es decir, usuarios que cuentan con un número elevado de seguidores y que por lo tanto se pueden articular como medios con audiencias en sí mismos. "Estos líderes de opinión del nuevo milenio empezaron su andadura como consumidores que compartían sus experiencias y daban consejos acerca de una variedad de productos mediáticos, como pueden ser videojuegos, series de televisión, libros, ropa, cosméticos o comida" (Establés et al, 2019).

El objeto del presente trabajo consiste en ir un paso más, examinar la construcción por parte de las agencias de comunicación y publicidad de no solo de una estrategia para promocionar las marcas o productos de sus clientes, sino la construcción misma de un influencer, en este caso virtual, al que poder dotar de atractivo y actividad lo suficientemente seductora como para conseguir un nivel de seguidores interesante en términos publicitarios. En muchas ocasiones, algunos de los seguidores de las marcas pasan usuarios participativos al hábito profesional de las industrias culturas, convirtiéndose en un fenómeno de la convergencia cultural (Jenkins, 2006). Las posibilidades de contar con un perfil de estas características son evidentes pues el control sobre sus comportamientos, eventos a los que acude, formas de expresión, etc... es total. En definitiva, el influencer virtual consigue aunar las ventajas de contar con un influencer tradicional en términos de notoriedad, atractivo y prescripción pero elimina los riesgos consustanciales a poner la marca, imagen y reputación en manos de una tercera persona.

El primer influencer virtual que aparece en las redes sociales data de hace tan solo cinco años. Desde entonces, la proliferación de este tipo avatares se ha convertido en un fenómeno recurrente. La buena acogida entre los usuarios reales de las diferentes redes sociales, así como sus enormes potencialidades en el plano comercial y social han favorecido los esfuerzos desde diferentes tipos de corporaciones por contar con una herramienta comunicativa diferencial, estratégica, rentable y sumamente dinámica. Pronto se pudo comprobar el enorme atractivo que para diferentes marcas comerciales de los sectores más variopintos generaban este tipo de perfiles y el negocio de publicidad y patrocinio que son capaces aglutinar. Además, fruto de una actividad que imita a los influencers de carne y hueso, los virtuales también muestran interrelaciones con usuarios reales y virtuales, adoptan posicionamientos políticos, muestran preocupación por causas y problemáticas socioculturales y pueden desempeñar un cierto activismo.

\subsection{Redes sociales. Evolución y características}

Para poder entender el fenómeno de los influencers como líderes de opinión del siglo XXI es necesario entender el funcionamiento de las redes sociales desde un punto de vista sociológico. En ese sentido, podemos afirmar que las redes sociales son: Individuos o grupos conectados por algún tipo de vínculo en común, estatus social compartido, funciones similares o compartidas, o algún tipo de conexión cultural o geográfica. Las redes sociales se forman o desaparecen dependiendo de las necesidades e intereses específicos de sus miembros (Barker, 1999, p. 399). 
De esta definición se desprende que el elemento fundamental para considerar a un grupo de personas como una red social es poseer un vínculo en común que mantenga a todos sus miembros unidos, pueden ser las relaciones de amistad, compartir la misma ideología, poseer un sentimiento patriótico o cualquier otra ocupación o entretenimiento. En un entorno físico, estos vínculos que mantienen a la red unida suelen ser tangibles, cohesionados y con un contacto presencial entre sus miembros. Sin embargo, Internet introduce la posibilidad de poder contar con otros miembros, que posean características parecidas en intereses similares a las personas que forman la red, pudiendo pasar a forma parte de esa red manteniendo vivos los lazos de unión durante un largo periodo de tiempo, sin necesidad de tener contacto presencial. En estos casos los vínculos se vuelven más indefinidos e intangibles en el mundo online; las conexiones se hacen más abiertas y las barreras entre grupos o comunidades se diluyen.

De todos es conocido que con la llegada de Internet se produce una nueva forma de comunicación entre las personas, unificando la comunicación interpersonal entre individuos con la capacidad de poder expandir el mensaje de un solo emisor a muchos sujetos al mismo tiempo, igual que hacen los medios de comunicación de masas. Esta nueva forma de comunicación fue denominada por Cathcart y Gumpert (1986) como comunicación interpersonal mediada o comunicación mediada por ordenador, y queda definida como "cualquier interacción persona-persona donde un medio de comunicación ha sido interpuesto para trascender las limitaciones del tiempo y el espacio".

Se produce, por tanto, un fenómeno nuevo donde las redes sociales interactivas permiten crear conexiones entre los sujetos en un espacio de comunicación masivo como ningún otro medio lo había permitido hasta el momento. Por este motivo, las redes sociales interactivas tienen otras características distintas a las redes sociales tradicionales. En términos generales, podemos afirmar que las redes sociales tradicionales tienen vínculos fuertes y muy cohesionados que las unen. Internet permite aumentar el número de vínculos entre los individuos de la red, comparándolo con una red social tradicional, al mismo tiempo que provoca que sean vínculos mucho más débiles y superficiales: por ejemplo, el compromiso que se adquiere asistiendo a un mitin político regularmente o a un concierto de un cantante no es equiparable a seguir a la página del partido político o la página del cantante en Facebook o compartiendo un video de YouTube.

Las redes sociales interactivas se conforman por pequeñas comunicaciones interpersonales que se establecen entre los individuos de la red, más que por una ideología o vínculo grupal.

La diferencia fundamental consiste en que estas comunicaciones son abiertas al público, elaborando mensajes públicos interpersonales (Walther et al., 2011). Esta nueva realidad en la que los conjuntos de individuos están conectados en un espacio abierto, unido por conexiones débiles y con mensajes privados abiertos a toda la red, genera un nuevo tipo de espacio de público que Danah Boyd (2011) denomina networked publics o públicos conectados. El concepto de público, con el significado de grupo de personas con un vínculo en común, ya se había utilizado con anterioridad al tratar grupos sociales y su vinculación con los medios de comunicación. Autores como Habermas (1989) ya habían utilizado el concepto de esfera pública para denominar un espacio en el que los miembros de la sociedad interactúan e intercambian información. Sonia Livingstone $(2005$, p. 9) también había empleado el concepto de público como "grupo de personas que comparten una misma manera de entender el mundo, una misma identidad colectiva o consenso a lo que respecta el interés colectivo". En el entorno online, el concepto de público adquiere otra significación. Estos públicos conectados quedan definidos según Boyd (2011) como públicos que están reestructurados por tecnologías de conexiones de redes. Por lo tanto, son simultáneamente:

- Espacio construido a través de estas tecnologías conectadas.

- Colectivo imaginario que emerge como resultado de la intersección de gente, 
tecnología y práctica.

Contemplan varias de las mismas funciones que tienen otros tipos de públicos: permiten reunirse por razones sociales, culturales o civiles y ayudan a conectar personas con un entorno más allá de sus amigos cercanos y familiares.

Mientras que estos "públicos conectados" comparten muchas características de otros tipos de grupos sociales, la manera en la que la tecnología los estructura introduce diferentes usos y posibilidades que cambia la manera de interactuar las personas en estos contextos tecnológicos. Esto trae consigo diferentes y nuevas dinámicas de participación personal.

Una vez enmarcado el entorno en el que se desarrollan las redes sociales digitales, estamos en condiciones de poder establecer ya una definición de estas. Para ello tomamos la de Boyd y Ellison (2007, p. 221) que definen las redes sociales como: servicios de Internet que permiten a los individuos:

- Construir un perfil público o semi-público dentro de un sistema cerrado.

- Articular una lista de otros usuarios con los cuales compartes una conexión.

- Ver y atravesar sus listas de conexiones y las de los otros usuarios que están dentro del sistema.

La definición de Ponce (2012, p. 16) pone el énfasis en señalar la función de interacción de las redes sociales digitales: Así se puede definir las redes sociales online como estructuras sociales compuestas por un grupo de personas que comparten un interés común, relación o actividad a través de Internet, donde tienen lugar los encuentros sociales y se muestran las preferencias de consumo de información mediante la comunicación en tiempo real, aunque también puede darse la comunicación diferida.

Esta definición pone de manifiesto la flexibilidad temporal de las relaciones interpersonales mantenidas en las redes sociales. No solo son tanto privadas como públicas, sino que pueden ser en tiempo real o diferidas, generando una sensación de superficialidad e indefinición de los vínculos que unen a esas personas. Además, los mensajes en estas redes pueden ser grabados, archivados y difundidos por otros, por lo que son mucho más persistentes, ya que se mantienen en el tiempo, aunque las circunstancias de esa persona cambien y la autoría o el iniciador de la conversación se haya diluido en el hilo de la conversación (Boyd y Ellison, 2007).

\subsubsection{Evolución de las redes sociales}

Para el estudio de la evolución de las redes sociales podemos distinguir cinco periodos diferenciados:

$1^{\text {o }}$ Primera aparición de las redes sociales (1997/2001)

La primera plataforma con las características propias de una red social fue la web SixDegrees.com (Boyd y Ellison, 2007). Esta red social permitía a los usuarios crear perfiles públicos, hacer listas de amigos y realizar búsquedas de otros amigos. Estas opciones ya existían por separado anteriormente en chats, foros o páginas webs de citas, pero Sixdegrees.com fue el primer servicio en aglutinar todos estos elementos. Esto provocó un cambio que definiría el curso de las redes sociales: la creación de perfiles fieles a la identidad offline.

A finales de los 90 comienzan a surgir redes sociales en distintos lugares del mundo y con diferentes finalidades como Asian Avenue, Blackplanet, Migente o Livejournal. Asimismo, otras plataformas cambiaron su modelo de negocio al de las redes sociales al ver la creciente popularidad como la sueca LunarStorm en 2001 o la página coreana Cyworld en 2000 (Boyd y Ellison, 2007). 
$2^{\circ}$ Las primeras redes sociales (2001/2004)

Las redes sociales comienzan a afianzarse con propuestas más definidas, enfocadas a públicos más concretos y con mayores funcionalidades para los usuarios.

La creación de Ryze.com en 2001 como plataforma para que las empresas pudieran crear sus propias redes sociales propició el desarrollo de nuevas redes sociales a partir de 2003, tanto de carácter profesional como LinkedIn o Xing como especializadas en un tema concreto.

Otras redes destacadas son:

- Friendster: considerada como la predecesora de Facebook y la primera red social creada como tal, para conectar amigos de amigos y así recomendar o vincular a pretendientes entre sí.

- MySpace: fundada en 2003 como una alternativa a Friendster, que en esos momentos perdía popularidad. MySpace no contaba con un target específico, pero la adopción de esta red por parte de bandas Indie-Rock hizo que MySpace empezara a posicionarse y promocionarse como servicio para grupos musicales.

$3^{\circ}$ Consolidación de las redes sociales (2004/2009)

En el año 2004 aparece la Web 2.0. Este hecho provocó la aparición de nuevas redes sociales. La consolidación de Internet en los hogares de todo el mundo permitió que las redes sociales consiguieran un alcance global y captaran audiencias masivas.

La aparición de negocios y páginas 2.0, con más contenidos interactivos y bidireccionales, propició el ambiente idóneo para la estabilización de las redes sociales. No solo las páginas webs incorporaron más elementos sociales y de interacción con el usuario, como recomendaciones o comentarios de otros usuarios, sino que negocios basados en contenidos generados por los propios usuarios, como Flickr o Last.FM, tenían características o rasgos propios de las redes sociales. Estos negocios también crecieron masivamente, creando una nueva cultura entre los jóvenes de compartir e intercambiar información en Internet entre sus redes de amigos o conocidos (Boyd y Ellison, 2007).

La aparición de Facebook fue sin duda la plataforma que cambió el curso de la evolución de las redes sociales. Facebook nació en 2004 como una red exclusiva para los estudiantes de Harvard, ya que solo se podía ser miembro a través del correo electrónico harvard.edu (Cassidy, 2006). En marzo de 2004, comenzó a extenderse a otras universidades de prestigio como Stanford, Columbia y Yale. En pocos meses llegó a otras muchas universidades como Boston University, New York Universit. Al año siguiente, Facebook se expandió también por los institutos de Estados Unidos y todas sus universidades. En 2006, empezó a expandirse a otros países. Durante 2007 y 2008 Facebook se adaptó a diferentes lenguas para llegar a países de habla no inglesa.

En este periodo, otras redes sociales globales comenzaron también su desarrollo y expansión. Twitter nació en 2006 como respuesta y/o evolución de los blogs. Se posicionó como herramienta para difundir noticias, contenidos o información en 144 caracteres de manera rápida, un formato totalmente insólito para la época. Por su parte, YouTube, que nació en 2005, revolucionó el contenido audiovisual a través de Internet.

$4^{\text {o }}$ Estabilización de las redes sociales (2009/2017)

Es en este periodo cuando podemos hablar de un momento de madurez de las redes sociales., Facebook se consolida como la red social más utilizada a nivel mundial. En abril de 2008, 
Facebook desbancó en número de usuarios activos mensuales a MySpace, debido a la consolidación de su botón "Me gusta" en el entorno web y a obtener beneficios gracias a su sistema de publicidad para empresas.

$5^{\circ}$ Las redes sociales en la actualidad (2017/2021)

La innovación tecnológica en las últimas décadas se situaba en los mercados asiáticos. Las seis, de las quince redes sociales con mayor número de usuarios se sitúan en Asia (Vicent, 2020) y dos de ellas tienen un uso a nivel mundial: WeChat y TikTok.

WeChat se ha convertido en una red social indispensable para cualquier empresa que quiera vender sus productos online en Asia.

También cabe destacar Tik Tok a la que la mayoría de los expertos apuntan como la próxima red social global. Esta plataforma nace en 2016 en China con el nombre de Douyin. Comenzó su expansión internacional al comprar la red social Musical.ly, enfocada a un público adolescente occidental, en la que los usuarios realizan videos con sus coreografías de canciones famosas (Yubal, 2017). En menos de dos años, Musical.ly aceleró su expansión, siendo la cuarta aplicación más descargada en España en 2019 (Solís Lerici, 2019). Sin embargo, va a se a finales de 2019 y primeros meses de 2020 cuando esta red pasa de ser un canal enfocado al público infantil y juvenil, a convertirse en un canal cada vez más masivo y utilizado también por el público adulto. En febrero de 2020 esta aplicación bate el récord de descargas a nivel mundial (Marketing Directo, 2020a) y con la crisis sanitaria del coronavirus se ha acentuado el traspaso masivo de usuarios de Instagram a Tik Tok.

\subsubsection{Las redes sociales en la actualidad en España}

Las redes sociales en España han alcanzado su madurez. El perfil de los usuarios de estas plataformas en España está muy repartido en la población: un $31 \%$ de los usuarios tienen de 16 a 30 años, un $39 \%$ de 31 a 45 años y un $30 \%$ de 46 a 65 años (IAB y Elogia, 2019), aunque estos datos no muestran el uso de los menores en estas plataformas, que previsiblemente será mucho mayor que los datos expuestos. Facebook es la red social que los españoles recuerdan más de manera espontánea con un $94 \%$, muy por encima de Instagram (69\%), Twitter (68\%) o WhatsApp (25\%) (IAB y Elogia, 2019). Sin embargo, cuando a los mismos encuestados se les sugiere un listado de redes sociales, Facebook se mantiene en primera posición con un $96 \%$ de conocimiento; aun así, goza de un conocimiento similar a otras redes como WhatsApp (93\%), Twitter (93\%), Instagram (84\%) y YouTube (82\%) (IAB y Elogia, 2019).

\subsubsection{El papel de los influencers}

La utilización de personajes virtuales que se convierten en influencers en las redes sociales ha propiciado el natural interés de las agencias publicitaria para contratar sus servicios al objeto de promocionar diversas marcas y productos, así se deduce de los estudios de Cillo y Prandelli (2020) que a su vez plantean interesantes reflexiones sobre cuestiones publicitarias clásicas como la transparencia en la prescripción de los productos, la pertinencia de determinados emplazamientos o circunstancias de entidad más ética y moral como la reproducción de determinados cánones estéticos.

Vázquez, Rodríguez Hernández y Fernández Fernández (2019) reflexionan sobre la importancia de la figura del influencer virtual en el mercado del lujo. Goikoetxea (2019) investiga la influencia de la inteligencia virtual en el desarrollo del sector moda. También podemos encontrar algunos estudios 
referidos al caso concreto de la más exitosa influencer virtual, Lil Miquela, Shieber (2019) que también servirá de base a nuestro estudio.

\subsection{Objetivos}

Con el fin de entender el papel de los influencers virtuales como elemento característico y definidor de las nuevas formas de consumo en las redes sociales se plantean los siguientes objetivos generales:

- Definir el concepto de los influencers en las redes sociales.

- Conocer el fenómeno de los influencers como mediadores y catalizadores del consumo.

De estos objetivos generales, se derivan los siguientes objetivos específicos:

- Diferenciar entre influencers reales y virtuales.

- Identificar el comportamiento que llevan a cabo los influencers virtuales y cómo se refleja en los mensajes comerciales que publican en sus redes.

- Analizar los mensajes generadores por los Lil Miquela en relación con la actividad comercial

- Explicar el éxito de los influencers virtuales en relación con el número de seguidores.

\section{Metodología}

Abordar un objeto de estudio novedoso como es el de los influencers virtuales en el que confluyen diferentes tecnologías punteras, novedosas formas de relación social y algunas cuestiones de corte ético y moral tradicional no es una cuestión sencilla. Consideramos pertinente señalar en este punto una serie de complicaciones iniciales que debemos resolver si no queremos caer en una excesiva dispersión que dificulte la obtención de resultados significativos:

- Dificultad a la hora de delimitar un objeto de estudio tan amplio y con tantas realidades.

- Las expectativas que genera, tanto de descripción de un proceso tan complejo como de explicación de su funcionamiento.

- La multiplicidad de sus ámbitos de aplicación.

Analizadas esas dificultades, el objeto de estudio queda centrado en la actividad comercial, entendiendo por contenido publicitario tres supuestos: la aparición explícita de la marca, el discurso sobre la marca y consumo de algún producto en el que la marca es claramente identificable en el caso de los avatares. Por tanto, el objeto de estudio se centra en los influencers virtuales y su capacidad de presentarse como imagen de marcas y prescriptores de productos, tomando como referencia el perfil de Lil Miquela, por tratarse de la influencer virtual con más seguidores en Instagram.

El marco temporal del estudio se circunscribe a lo largo de los últimos cuatro meses (septiembre, octubre, noviembre y diciembre) del año 2020.

La hipótesis que guía nuestra investigación quedo planteada en los siguientes términos:

Los influencers virtuales son importantes herramientas comerciales especialmente entre el público más joven, capaces de movilizar a este colectivo para obtener su deseo de compra, ya sea hacia un producto, marca o incluso estilo de vida.

Para llevar a cabo esta investigación, se ha planteado una metodología dividida en tres fases en las que se combinan técnicas cualitativas y cuantitativas a través de las cuales se puede realizar, en primer lugar un análisis de la muestra seleccionada, para poder llevar a cabo después el análisis de esos elementos mediante la observación y la aplicación de instrumentos de medición del impacto de 
las publicaciones y del contenido de estas (ver Tabla 1). Con la combinación de estas técnicas se pretende realizar un análisis profundo del comportamiento comercial mostrado por Lil Miquela.

Tabla 1. Metodología de la investigación

\begin{tabular}{|c|c|c|c|}
\hline Fase & Estudio & Tarea & Finalidad \\
\hline Primera & Teórico descriptivo & $\begin{array}{lrr}\text { Contextualización } & \text { y } \\
\text { revisión conceptual } & \text { del } \\
\text { fenómeno de } & \text { los } \\
\text { influencers } & & \end{array}$ & $\begin{array}{ll}\text { Estudio y reflexión de las } \\
\text { redes sociales y del } \\
\text { fenómeno de } & \text { los } \\
\text { influencers y de los } \\
\text { influencers virtuales }\end{array}$ \\
\hline Segunda & $\begin{array}{l}\text { Experimental } \\
\text { cuantitativo, cualitativo y } \\
\text { descriptivo }\end{array}$ & Trabajo experimental & $\begin{array}{l}\text { Elección de la muestra. } \\
\text { Análisis de contenido. } \\
\text { Análisis y resultados de la } \\
\text { muestra analizada }\end{array}$ \\
\hline Tercera & Conclusivo & $\begin{array}{llr}\text { Comprobar el } & \text { grado } & \text { de } \\
\text { cumplimiento } & \text { de } & \text { los } \\
\text { objetivos } & \text { de } & \text { la } \\
\text { investigación } & & \end{array}$ & $\begin{array}{l}\text { Comprobar el papel de las } \\
\text { redes sociales y de los } \\
\text { influencers virtuales como } \\
\text { catalizadores del consumo }\end{array}$ \\
\hline
\end{tabular}

Fuente: Elaboración propia.

En un primer acercamiento al objeto de estudio se han analizado las redes sociales y su evolución, así como el fenómeno de los influencers reales y los influencers virtuales. La segunda fase, está compuesta por un estudio experimental con el que se pretende analizar la muestra seleccionada desde un punto de vista cuantitativo y cualitativo. Para finalizar, una última fase conclusiva derivada del análisis realizado, en el que se comprobarán el grado de cumplimiento de la hipótesis y de los objetivos planteados.

Para la realización de esta investigación se ha seleccionado una muestra del perfil, con mayor número de seguidores, de la influencer virtual Lil Miquela. Se han analizado las 122 publicaciones realizadas en esta red social durante los últimos cuatro meses del año 2020, periodo posterior al confinamiento más duro de la pandemia sanitaria de la COVID-19. Dentro de las publicaciones se han investigado los distintos contenidos y las cuentas relacionadas con los diferentes posts.

\section{Discusión / Resultados}

\subsection{Redes sociales. Evolución y características}

Los denominados influencers se han convertido en los líderes de opinión del siglo XXI. En un momento en el que los contenidos televisivos se han quedado a un lado para dar paso a internet $\mathrm{y}$, más concretamente, a las redes sociales, la competencia por conseguir un mayor número de likes es el principal motor de la comunicación en estas plataformas. De esta manera, las marcas comerciales, apuestan por la figura de los influencers para dar a conocer su marca o lanzar sus mensajes publicitarios.

El estudio realizado por InfoAdex (2000) sobre la inversión publicitaria en España apuntaba que, en solo 25 años, la publicidad digital pasó de ser inexistente a convertirse en el medio con mayor inversión publicitaria en España en 2019, sobrepasando de esta manera a la televisión.

Ferrer López (2000) explicaba que el hecho que lleva a la población joven a estar presente en las redes sociales consiste en la necesidad de establecer conexiones y vínculos entre sus miembros. Una 
de las características principales de las redes sociales, es que todos los miembros pueden tener una participación en los distintos contenidos. Estas plataformas se han convertido en medios de comunicación bidireccionales dónde la opinión del público objetivo tiene una gran importancia.

Aunque en un primer momento se llegó a pensar que las redes sociales presentarían una dificultad considerable en las relaciones interpersonales, ha sucedido lo contrario. Así, numerosos estudios manifiestan que estas plataformas favorecen los vínculos interpersonales consiguiendo una mayor capacidad comunicativa entre los seres humanos incluso estando separados territorialmente (Birnie y Horvath, 2006; Ploderer et al., 2008).

La evolución de los medios de comunicación está íntimamente relacionada con el desarrollo tecnológico. La aparición y el gran desarrollo de internet han contribuido a transformar las relaciones humanas. En la obra del sociólogo Zygmunt Bauman, podemos observar:

1. En la actualidad, el concepto "de desplazamiento" ha desaparecido, sin emabrgo, en la antigüedad este concepto era totalmente indispensable para poder transmitir la información (Bauman, 1999, p. 24).

2. El desarrollo de los aparatos electrónicos, cada vez de menor tamaño, permiten convertirnos en emisores y receptores de mensajes abiertos, no solo a nuestros conocidos, sino también al resto de los navegantes en la red (Bauman, 2017, pp. 24-40).

3. El amplio desarrollo de la sociedad de consumo ha invadido todas las áreas de la vida humana. El desarrollo consumista ha llevado a aumentar la cantidad y la rapidez de las informaciones que se transmiten (Bauman y Donskis, 2015, pp. 56-61).

4. La aparición de las redes sociales permiten a los seres humanos vivir simultáneamente en dos mundos diferenciados: el tradicional, caracterizado por unas relaciones de pertenencia y el online, totalmente independiente y autónomo ya que la navegación por las redes sociales es libre y cada cual decide las informaciones que quiere recibir y las personas con las que quiere interactuar (Bauman, 2016, pp. 92-95).

En este mundo en el que vivimos, las marcas comerciales apuestan por crear contenidos participativos para "enganchar" a los consumidores más jóvenes. Se realiza una comunicación continua con la que se pretender conseguir un apego emocional e incondicional con sus seguidores.

Las posibilidades que actualmente presentan las redes sociales y la facilidad de conexión, en prácticamente cualquier lugar del mundo, hacen que los influencers se conviertan en un arma poderosa a la hora de generar contenidos relacionados con las marcas comerciales.

\subsection{Influencers Virtuales}

La importancia adquirida por las redes sociales como medio de comunicación y de relación con otros usuarios demuestra la importancia de este soporte publicitario, convirtiéndose en uno de los medios con mayor impacto e inversión del momento.

En 2016, gracias a la importancia que estaban adquiriendo los influencers en el mundo publicitario, se crea al primer influencer virtual. La razón fue crear un personaje con el que se pudieran controlar los mensajes así como su comportamiento. La principal ventaja de los avatares en redes sociales está en que ellos nacen y mueren en la red, y que detrás de ellos se encuentra un equipo formado para crear contenidos de interés y encapsular los mensajes publicitarios de la manera más adecuada. 
El principal objetivo de los influencers, tanto reales como virtuales, es captar la atención de sus seguidores y mantener su fidelidad. Instagram tiene una forma muy característica de poder contar historias, tal como explicaban Casas-Moreno, Tejedor-Calvo y Romero-Rodríguez:

Instagram se ha convertido en una plataforma para contar historias, relatando visualmente los actos de su vida cotidiana. Esta aplicación (Instagram stories) permite, además, añadir fotografías y vídeos cortos con la capacidad de agregar textos, emoticonos, filtros, etc. El cerebro humano está preparado para guardar la información a modo de relato. $(2018$, p. 45)

Los influencer virtuales han aumentado su éxito en los últimos cinco años, subiendo posiciones en relación con los influencer reales. Esto es debido al trabajo que las agencias de publicidad realizan en torno a estos perfiles. Un equipo especializado en comunicación es el encargado de crear los contenidos, trabajando, de forma muy estrecha, con directores de arte, de fotografía, realidad virtual y efectos especiales. De esta forma, se crean contenidos atractivos con capacidad para captar seguidores a través de un hilo conductor concreto.

Las personas que interactúan en redes sociales muestran intereses y preferencias sobre temas concretos. Esto es utilizado por los perfiles virtuales para crear historias que imiten esos intereses mostrando diferentes ámbitos de la vida cotidiana. Al estudiar a los seguidores se pueden elaborar mensajes y conductas sociales en los que las marcas tendrán un papel importante, estableciendo un vínculo de conexión entre los avatares y sus seguidores.

Otra de las ventajas de este tipo de perfiles es que se pueden realizar segmentaciones de la audiencia, consiguiendo posicionarse con un mayor número de seguidores. Esto se traduce en que su interés como plataforma de publicidad comercial aumenta, consiguiendo un mayor interés económico.

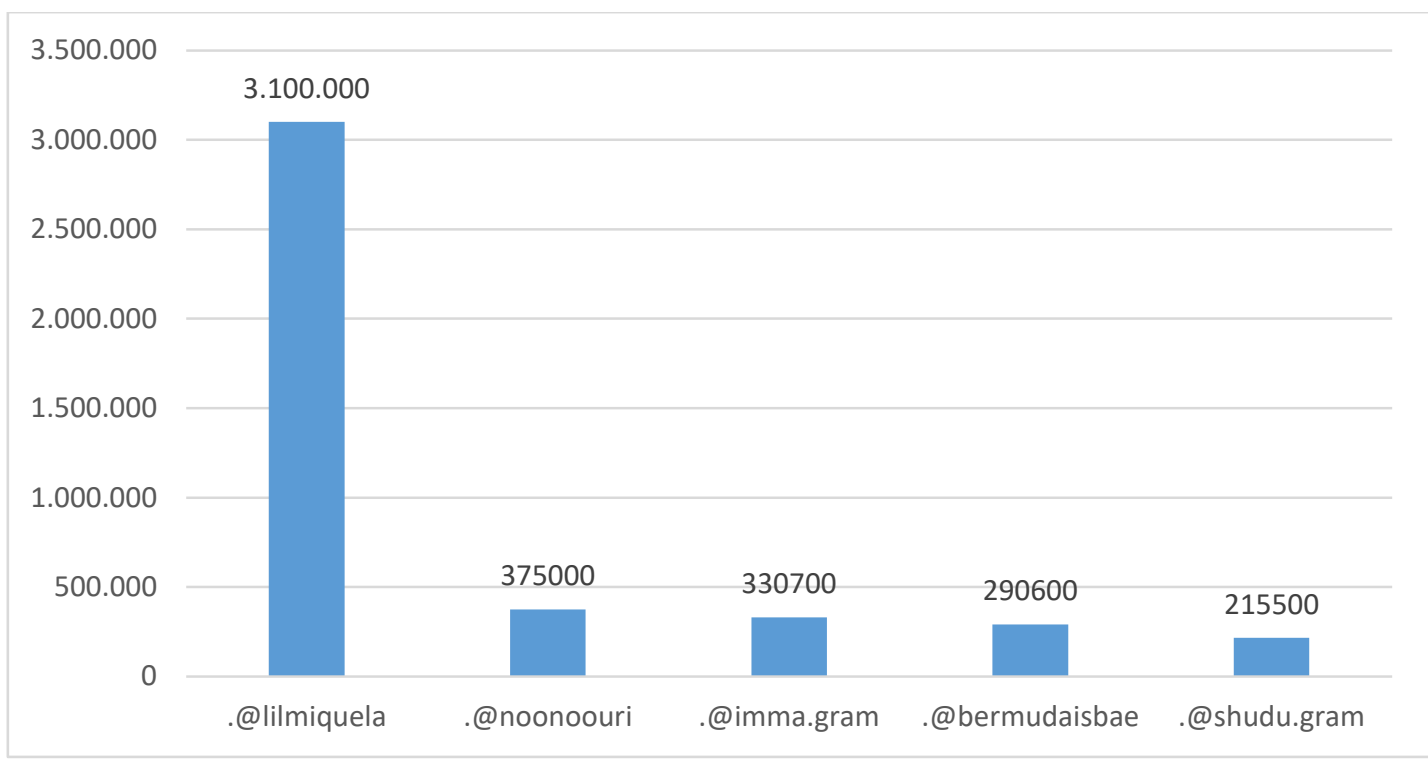

Gráfico 1: Número de seguidores de los cinco influencers virtuales más destacados

Fuente: HyperAuditor, 2021 
RLCS, Revista Latina de Comunicación Social, 79, 69-90

[Investigación] DOI: 10.4185/RLCS-2021-1521| ISSN 1138-5820| Año 2021

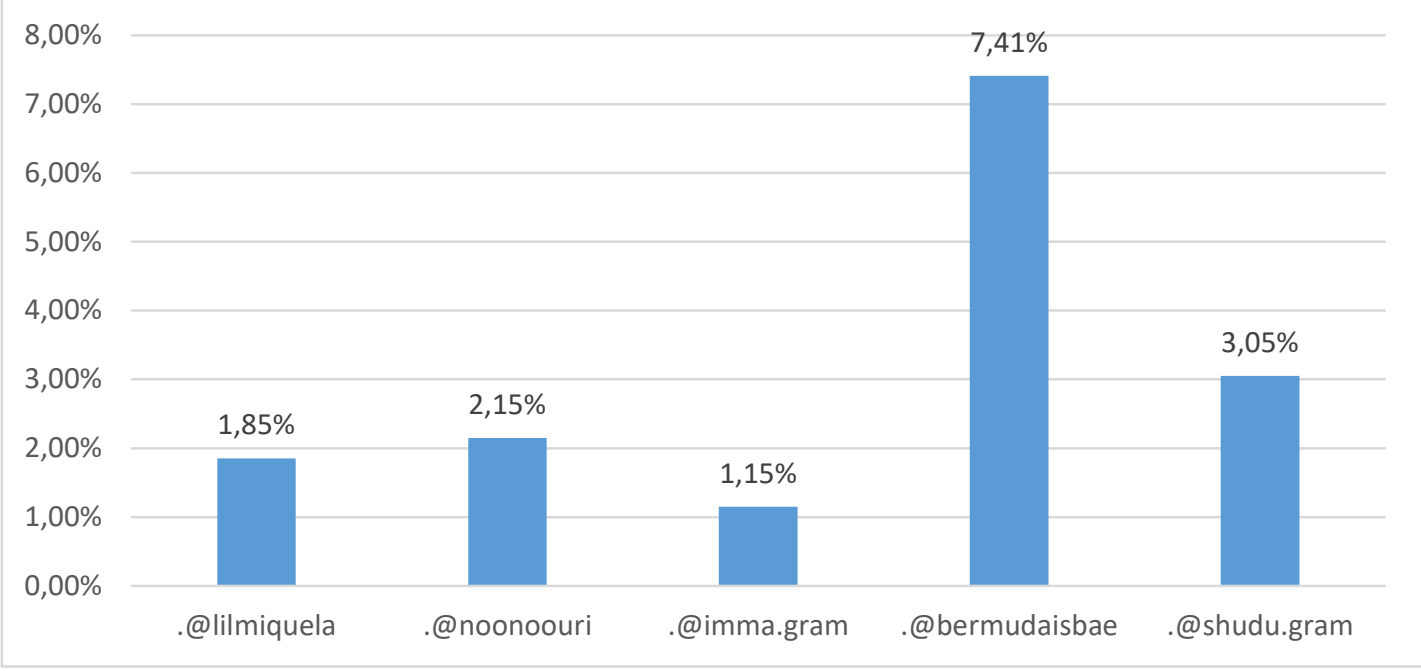

Gráfico 2: Porcentaje de los seguidores que da a "me gusta" o comenta publicaciones Fuente: HyperAuditor, 2021

En los gráficos 1 y 2 se puede observar una comparativa entre el número de seguidores de los perfiles de influencers virtuales más destacados y su porcentaje de la audiencia que comenta o da likes a las publicaciones (ER). La importancia marcada por el número de seguidores (más de 3.000.000 de seguidores) así como de los post publicados (1.080) hacen del perfil de Lil Miquela el más importante y el principal motivo por el que se haya elegido esta cuenta para la realización del trabajo de campo.

Por otro lado, podemos comprobar que el perfil más activo en relación con la interactuación con los seguidores es la cuenta de @bermudaisbae, sin embargo, en el último año este usuario no está realizando prácticamente publicaciones propias.

La firma Brund crea el 23 de abril de 2016 a la primera influencer virtual, Lil Miquela, que en la actualidad es el perfil de Instagram con mayor número de seguidores. Un robot, como se presenta en su cuenta, residente en Los Ángeles y que con sus eternos 19 años ha conseguido más de 3 millones de seguidores publicando un elevado número de post y de stories.

Este perfil ha convertido la vida de un avatar en el soporte perfecto para lanzar mensajes publicitarios, así como contenido social e ideológico.

El principal objetivo de estas cuentas es reflejar la realidad tal y como es, y ahí reside el éxito de los influencers virtuales. El mayor número de seguidores se encuentra entre la Generación Z, ya que, aunque saben que no son reales, tienen un alto grado de empatía con este tipo de perfiles. NúñezGómez, P., Rodrigo-Martín, L., Rodrigo-Martín, I. y Mañas-Viniegra, L. hablan de la necesidad de conocer las preferencias de este público objetivo para realizar mensajes con un mayor grado de profundización puesto que "Las marcas deberían investigar más a este nuevo público y dirigirse al mismo de una manera responsable y educativa, porque, a largo plazo, la fidelidad de la marca va a ir ligada a ello" (2020). Se intenta crear influencers virtuales lo más parecidos a sus seguidores para lograr una participación y efectiva. 


\subsection{Análisis de las publicaciones del perfil de Lil Miquela}

Para abordar el estudio del caso de Lil Miquela, realizamos un primer acercamiento a las publicaciones realizadas desde 2016. La afinidad y capacidad de influencia de este perfil con respecto al público objetivo puede verse reflejado en el aumento de las publicaciones realizadas a lo largo de estos años.

Tabla 2. Datos de las publicaciones realizadas por @lilmiquela

\begin{tabular}{|c|c|c|c|}
\hline Año & $\mathbf{N}^{\mathbf{~}}$ Post & Fecha Primero & Fecha Último \\
\hline $\mathbf{2 0 1 6}$ & 12 & 23 de abril & 28 de diciembre \\
\hline $\mathbf{2 0 1 7}$ & 55 & 27 de enero & 31 de diciembre \\
\hline $\mathbf{2 0 1 8}$ & 296 & 1 de enero & 31 de diciembre \\
\hline $\mathbf{2 0 1 9}$ & 273 & 2 de enero & 30 de diciembre \\
\hline $\mathbf{2 0 2 0}$ & 327 & 1 de enero & 30 de diciembre \\
\hline $\mathbf{2 0 2 1}$ & 103 & 1 de enero & 27 de mayo \\
\hline
\end{tabular}

Fuente: Elaboración propia.

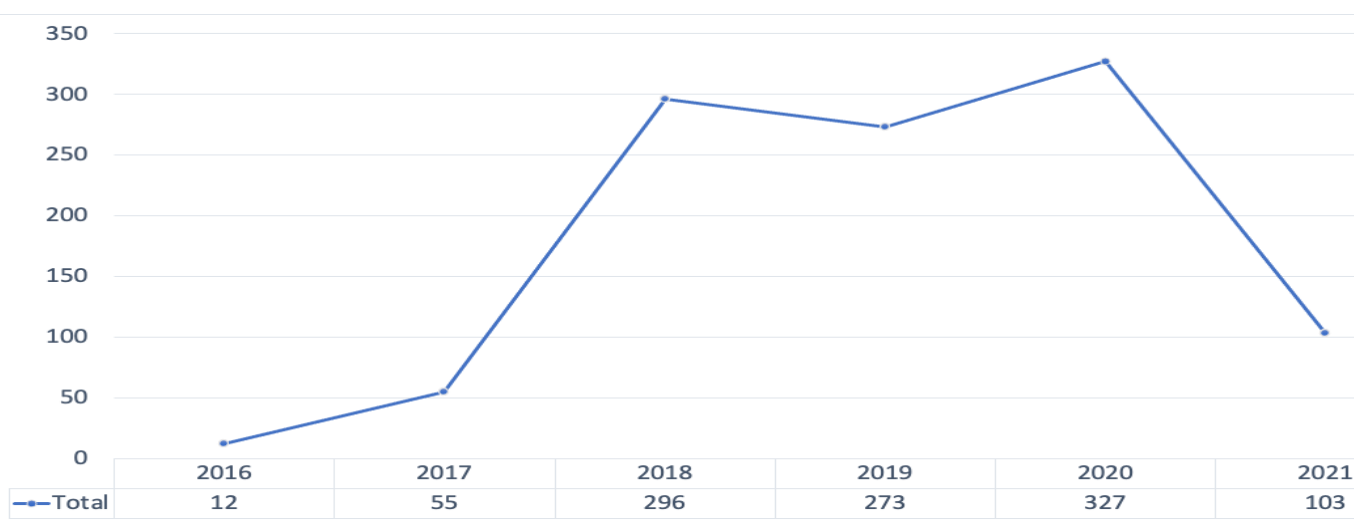

Gráfico 3: Evolución de publicaciones de @ lilmiquela

Fuente: Elaboración propia

La actividad realizada durante el primer año en el perfil de Lil Miquela, con 12 publicaciones, no es representativa. A partir de 2017 el perfil comienza a realizar un mayor número de publicaciones, aunque manteniendo una distancia clara con los influencers reales.

El mayor número de publicaciones se realizan durante los años 2018 y 2019 con casi 300 posts. Se comienza a lanzar mensajes muy cuidados y de forma natural, lo que hace que las grandes marcas de moda o lujo se decanten por la cuenta de Lil Miquela para soportar sus mensajes comerciales.

La crisis sanitaria debida a la COVID-19 hace que el 2020 se convierta en un punto de inflexión en los perfiles de los influencers virtuales. Estas cuentas no se vieron afectadas por las restricciones de movilidad o los confinamientos y siguieron mostrando la vida tal y como era antes del COVID. La búsqueda de la normalidad hizo que estas cuentas tuvieran un perfil alto de publicaciones durante el pasado año, aumentando significativamente en relación con años anteriores.

Desde el 1 de enero hasta el 27 de mayo de 2021, se han realizado 103 publicaciones, 14 publicaciones menos que en el mismo periodo de 2020. A pesar de que en estos momentos el número de publicaciones es menor, se sigue un crecimiento similar al de la etapa anterior. 


\subsection{Análisis de campo}

Para realizar el estudio se han seleccionado los cuatro últimos meses del año 2020, puesto que consideramos que es cuando comienza a normalizarse las relaciones sociales tras el periodo más duro de la pandemia sanitaria por la COVID-19. En total, se han analizado 122 publicaciones realizadas por la influencer virtual Lil Miquela.

El hecho de seleccionar un influencer virtual, el más seguido de la red social de Instagram con más de 3.000.000 de seguidores y 1080 publicaciones desde 2016, quiere demostrar que los gustos de los seguidores en Instagram no se basan en si el personaje es real o virtual, sino en su capacidad para entretener y fascinar. Por este motivo, los influencers virtuales están posicionándose rápidamente en el mercado, puesto que tienen un equipo detrás que trabaja por y para ellos, con el fin de crear espacios de entretenimiento, similares a las series de televisión, con la ventaja de que se puede jugar con el espacio y el tiempo sin costes adicionales.

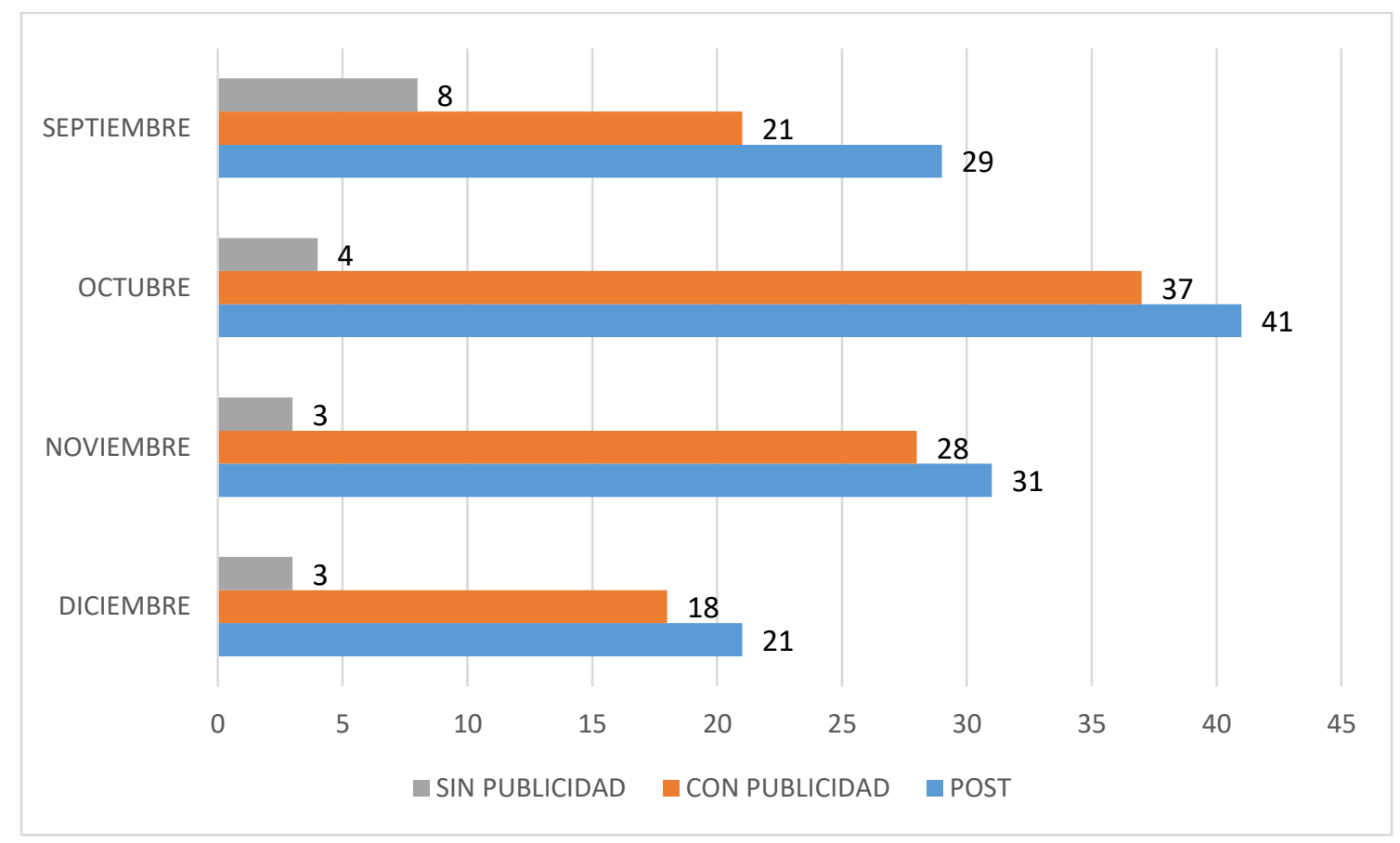

Gráfico 4: Contenido de los posts realizados por @lilmiquela

Fuente: Elaboración propia

Como se puede observar, la tendencia en publicaciones a lo largo de los meses analizados es muy similar, quedando por debajo de la media únicamente el mes de diciembre con 21 post. También encontramos dos tipos de publicaciones diferentes, unas con publicidad o referencia a marcas comerciales o eventos, y otras sin publicidad en las que se muestra el avatar realizando diferentes actividades de la vida cotidiana.

El $85 \%$ de las publicaciones analizadas tienen alguna referencia publicitaria, lo que hace de este perfil de Instagram un referente en publicidad comercial. Sin embargo, el $15 \%$ restante, al no contener referencias publicitarias intenta convertir a Lil Miquela, en un personaje amable, dando una sensación de normalidad e imitando a los influencers reales. 


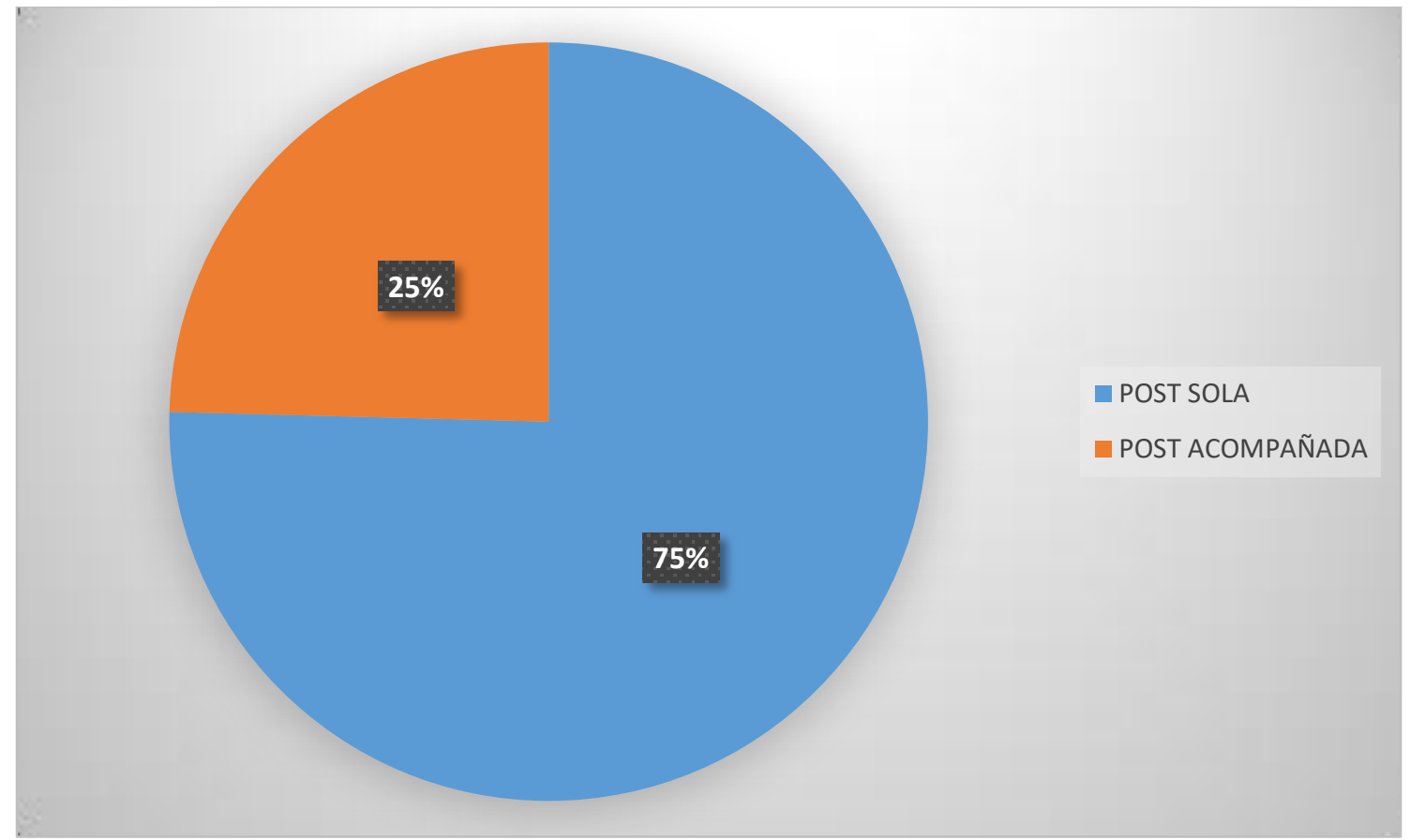

Gráfico 5: Porcentaje de publicaciones en las que Lil Miquela aparece en compañía de otras personas

Fuente: Elaboración propia

Otra de las principales características que tiene esta cuenta de Instagram es que es una cuenta personal. En la mayoría de las publicaciones realizadas aparece el avatar en solitario, solamente en el $25 \%$ de las publicaciones analizadas, podemos ver al avatar de Lil Miquela acompañada de otras personas, tanto reales como virtuales.

En el perfil de Lil Miquela se puede observar cómo también se realizan colaboraciones con otras cuentas. A lo largo de los post de los cuatro últimos meses de 2020, podemos ver a Lil Miquela acompañada de varios influencers virtuales y reales, lo que hace que esta cuenta adquiera una sensación de mayor realismo, puesto que se vincula un robot con las relaciones sociales que mantienen las personas reales. 


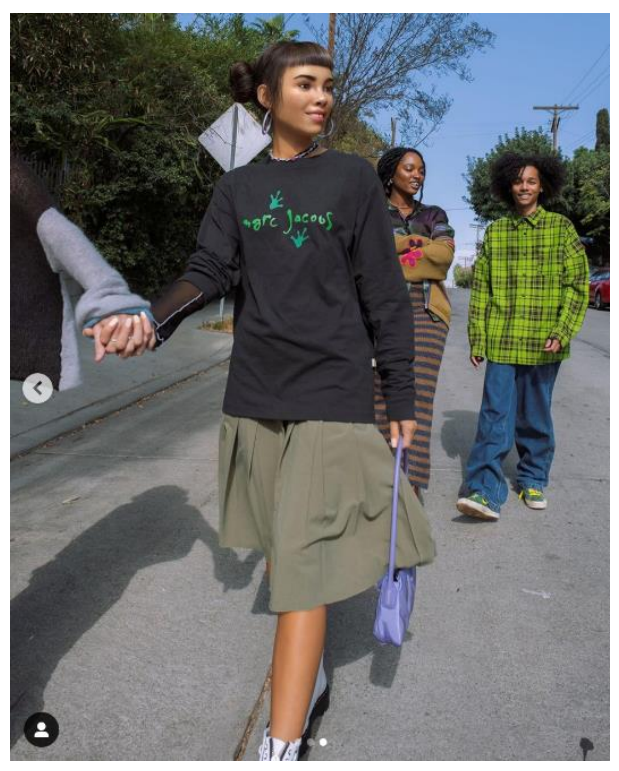

Imagen 1: Publicación del 28 de noviembre de 2020, en el que se muestran las relaciones sociales que mantiene Lil Miquela

Fuente: Perfil de Lil Miquela en Instagram, 2020

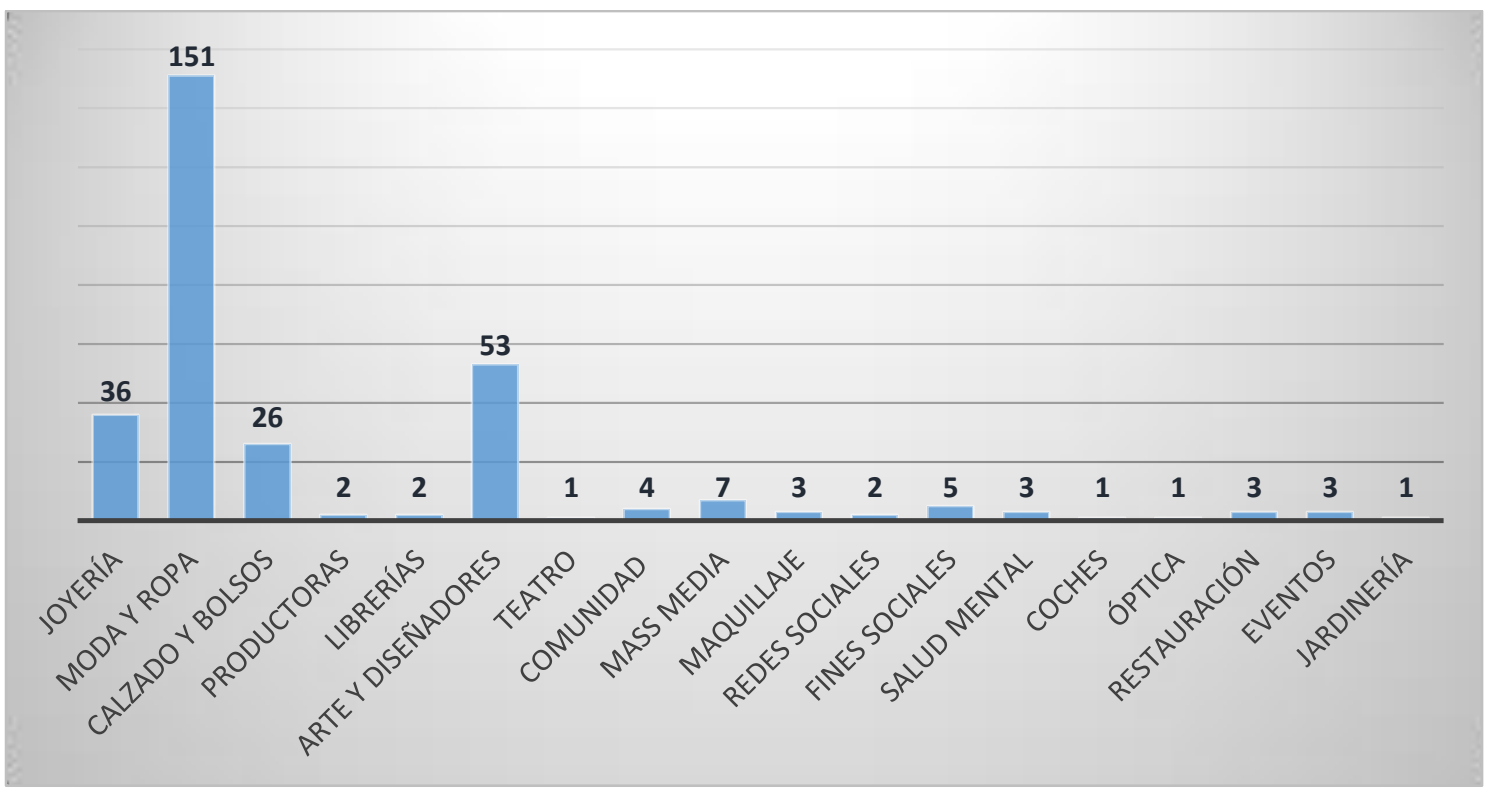

Gráfico 6: Porcentaje de los sectores anunciados en la cuenta @lilmiquela

Fuente: Elaboración propia

A lo largo del análisis se ha podido comprobar cómo los sectores que eligen la cuenta de @ lilmiquela para publicitar sus productos o servicios son variados, sin embargo, hay uno que destaca sobre los demás. El sector de la moda, concretamente en lo referido al textil, se coloca en primer lugar.

Un $50 \%$ de las publicaciones comerciales se realizan en torno al sector de la moda. En segundo lugar, pero con un porcentaje mucho menor, solamente el $17 \%$, se encuentra el sector relacionado con el arte, los artistas y los diseñadores. El $12 \%$ de las publicaciones realizadas giran en torno a la joyería y el $9 \%$ al sector de calzado y bolsos. 
Con este análisis podemos destacar que las publicaciones realizadas con un mayor porcentaje están muy relacionadas entre sí, puesto que tanto el calzado como la joyería o los diseñadores tienen un núcleo común como es la moda. De esta manera, Lil Miquela se ha convertido en un escaparate de marcas, todas ellas relacionadas con una modelo virtual, que en ocasiones también participa en pases de modelos y nos habla de sus gustos en función de qué marcas pagan más.

Otro de los aspectos destacados al analizar el perfil de Lil Miquela es que dentro de una misma publicación puede hacer referencia a varias marcas comerciales. Es decir, cuando publica un post relacionado con el sector moda, generalmente, hace referencia a varias marcas, especificando de qué marca es cada una de las prendas que lleva puestas, qué diseñadores las han realizado o dónde podemos encontrar las piezas de joyería que utiliza en una publicación determinada. Esto nos lleva a comprobar que, dentro de los 104 post con publicidad de marcas comerciales se realizan 151 referencias a moda y ropa, siendo, como se ha dicho, el sector más destacado en este perfil de Instagram.

Alejandra de la Riva (2017) apuntaba que las marcas de moda siempre pioneras en usar nuevas herramientas de comunicación, han sido las primeras en apostar por las nuevas tendencias. Un virtual influencer nos ayuda a llegar a un público más amplio y joven.

De esas 151 referencias a moda, encontramos 84 marcas diferentes entre las que se encuentran firmas de alta costura como Prada o Dior, pero donde el núcleo principal se centra en dar visibilidad a pequeñas empresas de moda o ropa ubicadas, generalmente, en EE. UU. Algo más de la mitad de las marcas comerciales, 44, solo son mencionadas una única vez, 40 dos veces, 14 tres veces, siete se citan cuatro veces, cinco otras tantas veces y la marca simonmillerusa aparece etiquetada en 6 publicaciones a lo largo de los últimos cuatro meses del año 2020, siendo la marca con mayor notoriedad en el periodo analizado.

Podemos decir que a pesar de que son muchas las marcas comerciales que utilizan este perfil de Instagram para darse a conocer, menos del $50 \%$ de esas marcas lo hacen de forma habitual. La gran notoriedad de esta cuenta, unido al alto número de seguidores hacen que aumente el coste de la publicidad.

\section{Conclusiones}

A lo largo del presente estudio se ha podido comprobar la gran capacidad de los perfiles creados para los influencer virtuales para constituirse como un soporte publicitario y de promoción de marcas comerciales de primer orden. Se ha demostrado que estos avatares desempeñan su función publicitaria en todos los supuestos planteados, en primer lugar, insertando la marca de una forma tradicional en sus publicaciones o post, en segundo lugar, construyendo un discurso en el que se cita y argumenta sobre la marca y, en tercer lugar, consumiendo productos de la marca, a modo de un emplazamiento de producto.

Consideramos que los objetivos planteados se han conseguido en un grado notable. El repaso por la historia de las redes sociales y sus mecanismos de funcionamiento e interrelación permiten trazar un perfil claro de la figura del influencer en términos generales y del influencer virtual en particular. Las diferencias entre uno y otro se limitan a su naturaleza puesto que en el plano comercial, objeto de este estudio, ambos operan de una forma semejante. Las ventajas de contar con un influencer virtual se derivan del control absoluto que el gestor de la cuenta tiene sobre el comportamiento y discurso del avatar lo que permite que se ajuste a la perfección a su labor comercial. Un influencer tradicional 
es una persona que toma decisiones propias y que lleva una vida que, en ocasiones, puede contradecir el discurso publicitario que sostiene en su actividad comercial.

La capacidad para generar atractivo, especialmente entre las personas más jóvenes de estos avatares, es un elemento que no ha pasado inadvertido en la industria publicitaria. Las agencias han encontrado en determinados perfiles un soporte publicitario válido para marcas de cualquier sector, principalmente de alto estatus y especialmente en el campo de la moda. El perfil tomado como base del estudio, Lil Miquela, publicita marcas y productos de los siguientes sectores: joyería, moda y textil, calzado y complementos, productoras, librerías, arte y diseñadores, teatro, comunidades, mass media, maquillaje, redes sociales, fines sociales, salud mental, automóviles, óptica, restauración, eventos y jardinería. Es decir, un abanico publicitario de primer orden, con resultados notables en todos esos campos.

El influencer virtual se convierte en una herramienta estratégica en tanto que no solo es capaz de promocionar marcas sino que, lo que es mucho más interesante, es capaz de generar tendencias. La construcción de un avatar que refleja un modelo y estilo de vida atractivo, sin restricciones de corte económico o jurídico, consigue conectar en el plano emocional con sus seguidores que experimentan algo parecido a una relación personal, por lo que su capacidad de prescripción se incrementa considerablemente fomentando que la actividad publicitaria aumente su efectividad.

El estudio de la actividad comercial de Lil Miquela ha permitido corroborar todos esos planteamientos previos. Su interés para marcas de diferentes sectores, su dinamismo a la hora de promocionar diferentes estilos de vida vinculados a marcas comerciales y su especial relación con audiencias jóvenes, convierten a los influencer virtuales en un elemento fundamental para entender la evolución de la actividad publicitaria en el actual contexto digital y para vislumbrar las tendencias de su evolución futura. La publicidad realizada a través de influencers virtuales tiene una capacidad de conexión con los públicos más jóvenes notablemente superior a otras fórmulas publicitarias y marketinianas de corte más tradicional lo que permite concluir que se convertirá en un elemento significativo de la publicidad de los próximos años.

\section{Referencias}

Barker, R. L. (1999). The social work dictionary (4th ed). NASW Press.

Bauman, Z. (1999). La globalización: Consecuencias humanas. Fondo de Cultura Económica.

Bauman, Z. (2005). La globalización. Fondo de Cultura Económica.

Bauman, Z. (2016). Extraños llamando a la puerta (A. Santos Mosquera, Trad.). Paidós.

Bauman, Z. (2017). Retrotopía (A. Santos Mosquera, Trad.). Paidós.

Bauman, Z. y Donskis, L. (2015). Ceguera moral: La pérdida de sensibilidad en la modernidad líquida (A. F. Rodríguez Esteban, Trad.; 1a. edición). Paidós.

Birnie, S. A. y Horvath, P. (2002). Psychological Predictors of Internet Social Communication. Journal of Computer-Mediated Communication, 7(4). https://doi.org/10.1111/j.1083$\underline{6101.2002 . t b 00154 . \mathrm{x}}$ 
Boyd, D. (2011). Social Network Sites as Networked Publics: Affordances, Dynamics, and Implications. En A networked self: Identity, community and culture on social network sites (pp. 39-58). Routledge.

Boyd, D. M. y Ellison, N. B. (2007). Social Network Sites: Definition, History, and Scholarship. Journal of Computer-Mediated Communication, 13(1), 210-230. https://doi.org/10.1111/j.1083$\underline{6101.2007 .00393 . x}$

Cassidy, J. (15 de mayo de 2006). Me media: How hanging out on the Internet became big business. The New Yorker, 82(13), 50-59.

Cillo, P. y Prandelli, E. (2020). A ogni campagna di marketing il suo influencer. Economia y management: la rivista della Scuola di Direzione Aziendale dell'Università L. Bocconi, 4, 75-80.

De Casas Moreno, P., Tejedor-Calvo, S. y Romero-Rodríguez, L. M. (2018). Micronarrativas en Instagram: Análisis del storytelling autobiográfico y de la proyección de identidades de los universitarios del ámbito de la comunicación. Revista Prisma Social, 20, 40-57.

Establés, M.J., Gerrero-Pico, M. \& Contreras-Espinosa R. (2019). Jugadores, escritores e influencers en redes sociales: procesos de profesionalización entre adolescentes. Revista Latina de Comunicación Social, 74, 214-236. https://doi.org/10.4185/RLCS-2019-1328

Fernández, Y. (10 de noviembre de 2017). Musical.ly es comprada por 1.000 millones de dólares, el valor de ser una app de moda entre adolescentes. Xataka.com. https://tinyurl.com/8j84dkk5

Ferrer López, M. (2020) Presencia de influencers en campañas publicitarias digitales. Estudio de su capacidad persuasiva y efectividad en jóvenes. [Tesis doctoral no publicada] Universidad Complutense de Madrid.

Florida Department of State. (2012). Third-Party Voter Registration Organizations. dos.myflorida.com. https://tinyurl.com/f6sd466j

Goikoetxea, J. (11 de marzo de 2021). Influencers virtuales, la guerra fría de la moda. Cactus. https://tinyurl.com/te4j65dk

González-Vallés, J.E., Barquero-Cabrero, J.D., Caldevilla-Domínguez, D. y Barrientos-Báez, A. (2021) Tipsters and addiction in Spain. Young people's perception of influencers on online sports Gambling. Int. J. Environ. Res. Public Health 2021, 18, 6152. https://doi.org/10.3390/ijerph18116152

Government of USA. (2021). Inscripción para votar en Estados Unidos. Usa.gov. https://www.usa.gov/espanol/inscripcion-para-votar

Gumpert, G. y Cathcart, R. S. (Eds.). (1986). Mediated interpersonal communication: Toward a newtypology. En Inter/media: Interpersonal communication in a media world (pp. 62-80). Oxford University Press.

Habermas, J. (1989). The structural transformation of the public sphere: An inquiry into a category of bourgeois society. MIT Press. 
HypeAuditor. (2021). Verifica al influencer antes de pagarle | Analíticas con IA de HypeAuditor para Youtube, TikTok e Instagram. HypeAuditor.com. Recuperado 16 de abril de 2021, de https://tinyurl.com/29stbfua

IAB Spain y Elogia. (2019). Estudio Anual de Redes Sociales 2019. https://tinyurl.com/336rvsc6

Infoadex. (2020). Estudio Infoadex de la inversión publicitaria en España, 2020. Infoadex, S.A. https://tinyurl.com/wwsm7fwv

Jenkins, H. (2006). Convergence Culture: Where old and New Media Collide. New York: NYU Press.

Miquela, L. [@lilmiquela]. (28 de noviembre de 2020). My processors were overloading, so I had to

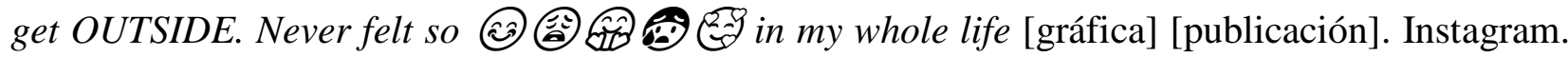
https://tinyurl.com/v784zu26

Livingstone, S. M. (Ed.). (2005). Audiences and publics: When cultural engagement matters for the public sphere. Intellect.

Marketing Directo. (16 de marzo de 2020). TikTok bate su propio récord en febrero con 113 millones de descargas. Marketingdirecto.com. https://tinyurl.com/npjk372j

Martínez Rodrigo, E. y Sánchez Martín, L. (2012). Comunicación entre menores y marcas en las redes sociales. Estudios sobre el Mensaje Periodístico, 18, 589-598. https://doi.org/10.5209/rev_ESMP.2012.v18.40938

Mañas-Viniegra, L. y Jiménez-Gómez, I. (2019) Evolución del perfil profesional del community manager durante la década 2009-2018. El profesional de la información, 28 (4), https://doi.org/10.3145/epi.2019.jul.03

National Voter Registration Day. (2020). About National Voter Registration Day | National Voter Registration Day. Recuperado 1 de febrero de 2021, de https://tinyurl.com/43jw6xxn

Núñez-Gómez, P., Rodrigo-Martín, L., Rodrigo Martín, I. y Mañas-Viniegra, L. (2020). Tendencias de Consumo y nuevos canales para el marketing en menores y adolescentes. La generación Alpha en España y su consumo tecnológico. Revista Ibérica de Sistemas e Tecnologias de Informação, E34, 391-407.

Pérez Curiel, C. y Clavijo Ferreira, L. (2017). Comunicación y Social Media en las Empresas de Moda. Revista Prisma Social, 18, 226-258.

Ploderer, B., Howard, S. y Thomas, P. (2008). Being online, living offline: The influence of social ties over the appropriation of social network sites. Proceedings of the ACM 2008 Conference on Computer Supported Cooperative Work - CSCW '08, 333-342. https://doi.org/10.1145/1460563.1460618

Ponce, I. (2012). Monográfico: Redes sociales. Observatorio Tecnológico. https://tinyurl.com/y29y643z 
Rodrigo Martín, I., Rodrigo Martín, L. y Muñoz Sastre, D. (2020). Storytelling como herramienta de promoción en redes sociales. Las redes sociales como herramienta de comunicación persuasiva, 2020, ISBN 978-84-486-2033-2, págs. 533-548, 533-548. https://tinyurl.com/h6u7yasr

Servimedia. (21 de junio de 2019). Crean el primer influencer virtual español. Diario de Sevilla. https://tinyurl.com/r8hrnh34

Shieber, J. (14 de enero de 2019). More investors are betting on virtual influencers like Lil Miquela | TechCrunch. Techcrunch.Com. https://tinyurl.com/5ypb5968

Solís Lerici, A. (1 de febrero de 2019). Así es Tik Tok: La cuarta app más descargada en España. Economiadigital.es. https://tinyurl.com/2mf8by92

Tur-Viñes, V., Núñez-Gómez, P. \& González-Río, M.J. (2018). Menores influyentes en YouTube. Un espacio para la responsabilidad. Revista Latina de Comunicación Social, 73, 1211-1230. https://doi.org/10.4185/RLCS-2018-1303

Vázquez Sacristán, I. A., Rodríguez Hernández, M. y Fernández Fernández, P. (2019). El fenómeno de los influencers virtuales en Instagram: Impacto y eficacia en el sector lujo. CUICIID 2019: Congreso universitario internacional sobre la comunicación en la profesión y en la Universidad de hoy IX: Contenidos, investigación, innovación y docencia: 23 y 24 octubre, 2019, ISBN 97884-09-17043-2, pág. 766, 766. https://tinyurl.com/chxm83k6

Vicent, J. (4 de febrero de 2020). Cuáles son las redes sociales con más usuarios en 2020. Trecebits.com. https://tinyurl.com/h8z8p259

Vilajoana-Alejandre, S., Rom-Rodríguez, J. y Miotto, G. (2019). Retos de la autorregulación publicitaria ante los riesgos jurídicos y éticos del marketing de influencers. Revista Mediterránea de Comunicación, 10(2), 115-129. https://doi.org/10.14198/MEDCOM2019.10.2.15

Walther, J. B., Carr, C., Choi, S., DeAndrea, D., Kim, J., Tong, S. y Van Der Heide, B. (2011). Interaction of interpersonal, peer, and media influence sources online: A research agenda for technology convergence. En A networked self: Identity, community and culture on social network sites (pp. 17-38). Routledge.

Watts, D. J. (2002). A simple model of global cascades on random networks. Proceedings of the National Academy of Sciences, 99(9), 5766-5771. https://doi.org/10.1073/pnas.082090499

\section{AUTORES:}

\section{Luis Rodrigo Martín}

Licenciado en Derecho (2002) y en Publicidad y RR.PP. (2003) por la Universidad Complutense de Madrid. Doctor en Comunicación Audiovisual y Publicidad por la Universidad de Valladolid con Premio Extraordinario de Doctorado (2010). Imparte docencia como Profesor Titular en la Facultad de Ciencias Sociales, Jurídicas y de la Comunicación de la Universidad de Valladolid desde 2003 en el grado en Publicidad y Relaciones Públicas en diferentes asignaturas vinculadas con el proceso creativo publicitario. Ha obtenido los premios de investigación Caja España y Caja Segovia. Autor de varios libros y una treintena de artículos en revistas científicas. Coordinador del Grupo de Investigación Icono 14 Tendencias en Publicidad y Consumo. 
luis.rodrigo@uva.es

Índice H: 7

Orcid ID: https://orcid.org/0000-0003-0580-9856

Google Scholar: https://scholar.google.es/citations?hl=esyuser=2g2SCg0AAAAJ

ResearchGate: AAC-8729-2019

Scopus ID: 56041393200

\section{Isabel Rodrigo Martín}

Licenciada en Publicidad y Relaciones Públicas por la Universidad de Valladolid en 2006. Doctora en Comunicación Audiovisual y Publicidad (Premio Extraordinario). Realizó el máster en Comunicación con fines sociales: estrategias y campañas, de la Universidad de Valladolid. En la actualidad es Profesora Titular de la Universidad de Valladolid. Obtuvo el Premio de Investigación Caja España 2007. Beca de Investigación de la Diputación de Segovia en 2016. Coordinadora del Proyecto de Innovación Docente: "Publicidad Social y Aprendizaje Servicio. Una experiencia compartida entre el alumnado universitario y el de educación primaria para la adquisición de una conciencia personal, social y ambiental para el progreso social" desde la convocatoria 2017/2018. Pertenece a la Cátedra de Comunicación Digital en Infancia y Adolescencia y al Grupo de Investigación SocMedia, UCM.

isabel.rodrigo@uva.es

\section{Índice H: 5}

Orcid ID: https://orcid.org/0000-0001-8349-5093

Google Scholar: https://scholar.google.es/citations?hl=es\&user=r94DrVcAAAAJ

ResearchGate: AAC-8051-2019

Scopus ID: 56041893300

\section{Daniel Muñoz Sastre}

Licenciado en Publicidad y Relaciones Públicas por la Universidad Complutense de Madrid (2000). Doctor en Comunicación Audiovisual y Publicidad por la Universidad de Valladolid (2015). Profesor de la Universidad de Valladolid donde imparte las asignaturas de Cultura e Identidad Corporativa y Gestión de Marca. Sus investigaciones están centradas en los retos de la transformación digital en el ámbito del corporate, y sobre la implantación del 5G. Es co-coordinador de un proyecto de innovación docente basado en el aprendizaje servicio entre alumnos de primaria y universitarios. Ha participado en actividades de transferencia de conocimiento llevadas a cabo con diversas empresas e instituciones. Además, ha sido redactor jefe en la Cadena SER y en la Cadena COPE.

daniel.munoz.sastre@uva.es

\section{Índice $\mathrm{H}: 4$}

Orcid ID: https://orcid.org/0000-0002-1136-5289

Google Scholar: https://scholar.google.es/citations?user=vbdJ-qQAAAAJ\&hl=es\&oi=ao

ResearchGate: Q-2882-2018

Scopus ID: 57191900962 